[CONTRIBUTION FROM THE JOHN HARRISON LABORATORY OF CHEMISTRY, No. 74.]

\title{
THE REDUCTION OF NITRIC ACID IN METALLIC NITRATES TO AMMONIA BY THE ELECTRIC CURRENT. ${ }^{1}$
}

BY WM. H. Fastox.

Received luly 6,1903 .

LUCKow announced" that "dilute nitric acid between two platinum poles will not be reduced to ammonia either alone or in the presence of dilute sulphuric acid. But, if copper sulphate in solution be added to the dilute nitric acid, then the formation of ammonium sulphate occurs, when the liquid is electrolyzed." Vortmann ${ }^{3}$ endeavored to make this a quantitative method for the estimation of nitric acid. ${ }^{4}$

This is his description of the analysis of a nitrate:

"The solution of the nitrate is placed in a platinum vessel with a sufficient amount of pure copper sulphate and it is then electrolyzed. It is advantageous to use a feeble current (I to $2 \mathrm{cc}$. of oxyhydrogen gas per minute). When all of the copper is deposited, the residual liquid is evaporated to a small bulk and the ammonia distilled off as usual."

Simple as these directions are, many persons have failed to get satisfactory results. Numerous inquiries have been made at this laboratory for fuller details; hence, the writer was directed to give the problem attention, more particularly with reference to the factors which have never before been studied in this particular example, viz., amperage, time, current density at the anode, the amount of copper sulphate essential, the amount of nitrate and the character of the cathode.

Potassium nitrate was the first nitrate analyzed. The conditions were:

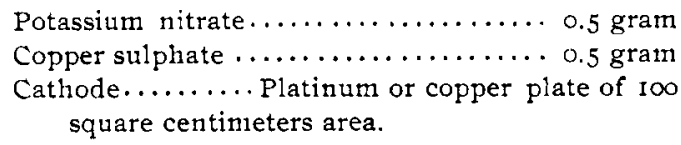

Amperes ...................... 5 to 3.0

Time ............ for 3.0 amperes, $I / 4$ hours

for 0.15 ampere, $81 / 2$ hours

'From the author's thesis for the Ph.D. degtee.

"Ztschr. anal. Chem., 19, i I (1880).

3 Ber. d. chem. Ges., I890, p. 2798.

"See Smith's "Electrochemical Analysis." p, i94, third edition, 1902. 
Volts: From 3 to 8 , depending upon the current strength.

Dilution: I $50 \mathrm{cc}$.

Sulphuric acid (sp. gr. I.062) : $30 \mathrm{cc}$.

The following percentages of nitrogen reduced to ammonia were obtained, the theoretical per cent. in potassium nitrate being I 3.86 :

$\begin{array}{llll}13.70 & 13.60 & 13.50 & 13.65 \\ 13.74 & \text { I } 3.68 & 13.77 & 13.54 \\ 13.62 & 13.43 & 13.32 & 13.68 \\ 13.43 & I 3.78 & 13.50 & \end{array}$

It was further learned, from additional experiments, that the time factor could be reduced to two and one-half hours by employing a current of I ampere. Further, in no trial did the variation of the current density at the anode have any influence on the amount of nitrogen reduced to ammonia. The amount of copper sulphate needed can easily be deduced from the results which follow, the other conditions being as recorded above.

\begin{tabular}{|c|c|c|}
\hline $\begin{array}{c}\text { Grams } \mathrm{KNO}_{3 .} . \\
0.5195\end{array}$ & $\begin{array}{c}\text { Granis } \mathrm{CuSO}_{4.5} \mathrm{H}_{2} \mathrm{O} \\
0.1098\end{array}$ & $\begin{array}{c}\text { Per cent. N reduced. } \\
\text { II. } 44\end{array}$ \\
\hline 0.5243 & $0.208_{3}$ & 12.23 \\
\hline $0.504 \mathrm{I}$ & 0.2600 & 13.33 \\
\hline 0.4950 & 0.3600 & 13.13 \\
\hline 0.5676 & $0.569 \mathrm{I}$ & I 3.74 \\
\hline
\end{tabular}

Theory, 13.86 per cent.

The quantity of potassium nitrate was also varied, equal weights of copper sulphate being taken, while the other conditions continued as before:

Grams of potassium nitrate.

0.1053

0.1063

0.2592

0. 2616

0.5027

0.5723
Grams of nitrogen
reduced.
O.OI 48

$0.014^{8}$

0.0348

0.0356

0.0693

0.0789
Per cent. of nitrogen

reduced.

I4.OI

I 3.93

I 3.43

I 3.62

I 3.77

I 3.78

The method seems accurate enough for all quantities. The actual weights of nitrogen show the delicacy of the method.

In the preceding experiments platinum dishes were the cathodes. The copper deposits were compact and readily washed. When copper plates of Ioo sq. cm. area were substituted for the dishes and suspended in the nitrate solution, contained in beakers, the 
precipitated copper was spongy, did not adhere well, and some difficulty was had in washing it. The percentages of reduced nitrogen ranged from I 3.04 to 13.44 (theory, 13.86 ). Lead plates of $100 \mathrm{sc} . \mathrm{cm}$. area were also tried. The copper was precipitated upon them in a very unsatisfactory form. They were also attacked, and the nitrate was never completely reduced when they were employed. No better success was met with mercury cathodes, while nickel $(36 \mathrm{sq} . \mathrm{cm}$. area) proved very satisfactory.

The time necessary for the reduction of 0.5 gram of potassium nitrate was two and one-half hours, when using a current of $I$ ampere. Would 3 or 5 amperes produce the desired result in a shorter period? Accordingly, a solution of 0.5009 gram of potassium nitrate, I gram of copper sulphate and $40 \mathrm{cc}$. of sulpluric acid (sp. gr. I.062) was electrolyzed for thirty minutes with a current of 3 amperes. The copper was not completely precipitated and only 6.68 per cent. of the nitrogen was reduced to ammonia. Upon increasing the current to 5 amperes and allowing it to act for thirty minutes the percentage of reduced nitrogen was I3.22, although not quite all of the copper was precipitated.

It was a matter of considerable interest to learn the behavior of other nitrates than those of the alkali metals. Copper nitrate electrolyzed alone-without copper sulphate-yielded II.52 and I I.35 per cent. of nitrogen (theory, II.59 per cent.) to the current. In the case of silver nitrate, complete reduction was not reached until after the addition of an equal amount of copper sulphate. This proved to be the case with cobalt and cadmium nitrates.

Observing the conditions given on p. IO42, employing at the same time a current of $N . D_{100}=\mathrm{I}$ ampere, the reduction will proceed rapidly and the method will prove very helpful in many instances.

Experiments have been made with a rotating anode which suggest the possibility of reducing quite considerably the time factor in this method.

Liviersity of Pennsylvania. 